\title{
Characteristics of Submeso Winds in the Stable Boundary Layer
}

\author{
Larry Mahrt
}

Received: 22 July 2008 / Accepted: 10 November 2008 / Published online: 28 November 2008

(C) The Author(s) 2008. This article is published with open access at Springerlink.com

\begin{abstract}
The characteristics of submeso motions in the stable boundary layer are examined using observations from networks of sonic anemometers with network sizes ranging from a few hundred metres to $100 \mathrm{~km}$. This study examines variations on time scales between $1 \mathrm{~min}$ and $1 \mathrm{~h}$. The analysis focuses on the behaviour of the spectra of the horizontal kinetic energy, the ratios of the three velocity variances, their kurtosis, the dependence of horizontal variability on time scale, and the inter-relationship between vertical vorticity, horizontal divergence and deformation. Motions on larger time and space scales in the stable boundary layer are found to be nearly two-dimensional horizontal modes although the ratio of the vorticity to the divergence is generally on the order of one and independent of scale. One exception is a small network where stronger horizontal divergence is forced by a decrease in surface roughness. The horizontal variability, averaged over $1 \mathrm{~h}$, appears to be strongly influenced by surface heterogeneity and increases with wind speed. In contrast, the time dependence of the horizontal structure on time scales less than one hour tends to be independent of wind speed for the present datasets. The spectra of the horizontal kinetic energy and the ratio of the crosswind velocity variance to the along-wind variance vary substantially between networks. This study was unable to isolate the cause of such differences. As a result, the basic behaviour of the submeso motions in the stable boundary layer cannot be generalized into a universal theory, at least not from existing data.
\end{abstract}

Keywords Mesoscale $\cdot$ Nocturnal boundary layer $\cdot$ Stable boundary layer $\cdot$ Submeso . Wind networks

\section{Introduction}

Compared to turbulence, relatively little is known about typical submeso/mesoscale motions in the stable boundary layer. The nocturnal mesoscale motions examined in this study extend

L. Mahrt (凶)

COAS Oregon State University, Corvallis, OR 97331, USA

e-mail: mahrt@nwra.com 
down to Eulerian time scales of a minute or less, horizontal length scales as small as $10 \mathrm{~m}$ and vertical depth scales as small as several metres. To reduce conflicts with traditional use of the term "mesoscale", we will use the term "submeso" to describe motions extending down to such small scales. The small-end of the mesoscale range in the ocean is often referred to as the sub-meso scale (see Fox-Kemper et al. 2008 and references therein), sometimes reserved for motions that are thinner than the boundary-layer depth. Mestayer and Anquetin (1995) defined "submeso" in the atmosphere within the context of dispersion in an urban setting associated with horizontal scales of $10-200 \mathrm{~m}$ in the lowest $20 \mathrm{~m}$. The model SUBMESO allows examinations of flows on scales smaller than $\mathrm{O}(\mathrm{km})$ where nonhydrostatic effects and noncomplicance with incompressible mass continuity may become important (Anquetin et al. 1998). The direct Coriolis effect is considered to be unimportant for submeso motions. Belušić and Mahrt (2008) referred to observations of nonturbulent motions on horizontal scales less than a few kilometres in the stable boundary layer as submeso. Unfortunately, the flow characteristics seem to vary continuously with scale, and the physics remains generally unknown.

In order to promote physical understanding, the literature has concentrated on infrequent cases of well-defined monotonic gravity waves, solitons, and drainage flows. Even then, the submeso motions in the stable boundary layer have been quantified primarily in terms of time variations observed from fixed towers. Based on numerical studies, laboratory experiments and theoretical considerations, the submeso modes in stratified flow are viewed as a combination of gravity waves and horizontal modes, sometimes referred to as pancake vortices (see, for example, Riley and Lelong 2000; Waite and Bartello 2004; Meunier et al. 2005, and references therein). Interaction between the gravity waves and two-dimensional modes are explored by Godoy-Diana et al. (2006), and interaction between turbulence, submeso motions and drainage flows was demonstrated by Monti et al. (2002). Horizontal modes have been difficult to identify from atmospheric data, perhaps due to inadequacy of the datasets. Even gravity waves involve a complex variety of generating mechanisms that includes overlying critical levels, reflection at the surface and ducting in the nocturnal boundary layer (Nappo 2002), as well as stationary waves associated with even shallow topography (Steeneveld et al. 2008). The latter is not captured by time series at a fixed point. Submeso motions in the stable boundary layer may assume a wide variety of other forms (see references in Mahrt 2007).

Recent networks of fast response sensors allow direct quantitative examination of the spatial structure of submeso motions near the surface. While such networks were not specifically designed for examination of submeso/mesoscale modes in the nocturnal boundary layer, they do reveal useful stochastic information on the submeso motions (Belušić and Mahrt 2008). The goal of this study is to examine the stochastic time-space structure of the submeso motions.

\section{Data}

The datasets analyzed here are described in more detail in Mahrt et al. (2008) and in the references listed in Table 1. I have applied only nominal additional quality control to the datasets to remove occasional spikes. For the analysis below, the time series of the wind components are divided into 1-h records.

The height of the sensors varies from $10 \mathrm{~m}$ in the Utah and Hereford networks to $5 \mathrm{~m}$ for the CASES99, EBEX and OHATS networks, $2 \mathrm{~m}$ above the crops for the Iowa network and $1 \mathrm{~m}$ above ground in the Young Pine and Mary's River networks. The Utah network (uncited) consists of 26 stations with R. M. Young Model 05103 anemometers with an accuracy of 
Table 1 Network name, surface conditions (sfc), the number of stations (stns), the domain width (km) defined as the maximum distance between stations $(\mathrm{km})$ and scales of computation of the gradients $(\mathrm{km})$ for those datasets analyzed in Sect. 2.2

\begin{tabular}{lllclll}
\hline Site & sfc & stns & Width & g. scale & Duration & References \\
\hline CASES99 & Grass & 7 & 0.60 & 0.4 & 4 weeks & Poulos et al. (2001) \\
EBEX & Cotton & 8 & 0.97 & & 1 month & Oncley et al. (2007) \\
Hereford & Grass & 13 & 13.9 & & 2 weeks & Hanna and Chang (1992) \\
Iowa & Crops & 9 & 10.2 & $1.8 / 5.4$ & 3 weeks & Kustas et al. (2004) \\
Mary's River & 20-m fir & 7 & 0.13 & & 4 months & Thomas et al. (2008) \\
OHATS & Sea & 9 & 0.005 & & 2 months & Sullivan et al. (2006) \\
Utah & Semi-arid & 26 & 100.5 & $7 / 21$ & 1 month & \\
Young Pine & 3-m pine & 9 & 0.21 & $0.04 / 0.12$ & 4 months & Schwarz et al. (2004) \\
\hline
\end{tabular}

The sixth column is the duration of the field program

$0.3 \mathrm{~m} \mathrm{~s}^{-1}$ and an official startup threshold of $1.0 \mathrm{~m} \mathrm{~s}^{-1}$. Surface vegetation consists primarily of sparse semi-arid grasses and bushes. The Iowa network will be chosen as the primary network since it is characterized by the best station configuration for the current analysis.

Variations of the wind between stations may be partly due to stationary horizontal variations in addition to transient spatial variability, examined in Mahrt et al. (2008) for these datasets. Stationary influences include surface heterogeneity and any instrument biases. While the Utah network resolves primarily scales larger than the submeso range and the Iowa and Hereford networks bridge submeso and mesoscale ranges, we will casually use the term submeso to collectively refer to all of the networks.

\subsection{Decomposition in Time}

Since the structures in the time series are generally local and not periodic, we orthogonally decompose the flow into the local multiresolution basis set (e.g., Howell and Mahrt 1997). Fourier decomposition into sinusoidal functions is most suitable for periodic phenomena such as monotonic gravity wave trains while events such as individual microfronts and solitions are more appropriately represented by local basis sets. Although no one basis set can be used to physically interpret the data, the scale dependence based on the multiresolution decomposition is generally similar to that based on the Fourier decomposition, although less noisy and often shifted to smaller scales. The multiresolution basis set corresponds to unweighted averages over windows of different dyadic widths and is the only basis set that satisfies Reynolds averaging independently at all scales. An arbitrary variable, $\phi$, for the ith record is written as

$$
\phi(i)=\frac{1}{M} \sum_{1}^{M} \phi_{m}(i)
$$

where $\phi_{m}(i)$ is the mth component of the decomposition. We will apply the decomposition to 1-min averaged data, corresponding to multiresolution dyadic time scales of 1, 2, 4, 8, 16, 32 and $64 \mathrm{~min}$. With this decomposition, the 1-min mode equates to the 1-min deviations from the 2-min averages, the 2-min mode is the deviation of 2-min averages from 4-min averages, and so forth. This decomposition requires 64-min records, which are constructed by overlapping 1-h records by $4 \mathrm{~min}$ in order that each record starts on the other. The overall conclusions are not affected by this overlap. The 1-min averaging was chosen based on the 
observation that the turbulent scales rarely exceed $1 \mathrm{~min}$ for stable conditions where the range of turbulent scales was determined as in Vickers and Mahrt (2006).

\subsection{Computation of Kinematic Quantities}

Kinematic quantities require evaluation of horizontal gradients. Toward this goal, the station data are mapped onto a regular grid with a grid spacing equal to about $10 \%$ of the network width. Data at the grid points are computed as a weighted average of the station data where the weighting function for each station is inverse-square proportional to the distance between the grid point and the station. This approach appears to be more stable than analytically fitting the wind fields. More complex interpolation techniques, such as krieging, did not appear to have any advantages over the simpler approach used here. A computational cross is defined as four of the grid points centered about a point in the approximate centre of the network. This grid cross is used to compute the gradients at the central point with simple centered differencing. This is the simplest configuration that allows computation of the gradients in the north-south and east-west directions. Gradients are computed only for records where all stations are available. For some networks, the gradients are computed on two different scales. The widths of the gradient computations are listed in Table 1, column 5.

\section{Dependence on Time Scale}

The spectra of the horizontal part of the kinetic energy, averaged over all of the nocturnal records in the Iowa data, reveals a well-defined peak at a time scale of about $7 \mathrm{~s}$ (Fig. 1). A partial gap between turbulence scales and submeso scales occurs at a time scale of a few minutes. The well-defined scale dependence in Fig. 1 breaks down for individual records with large nonstationarity.

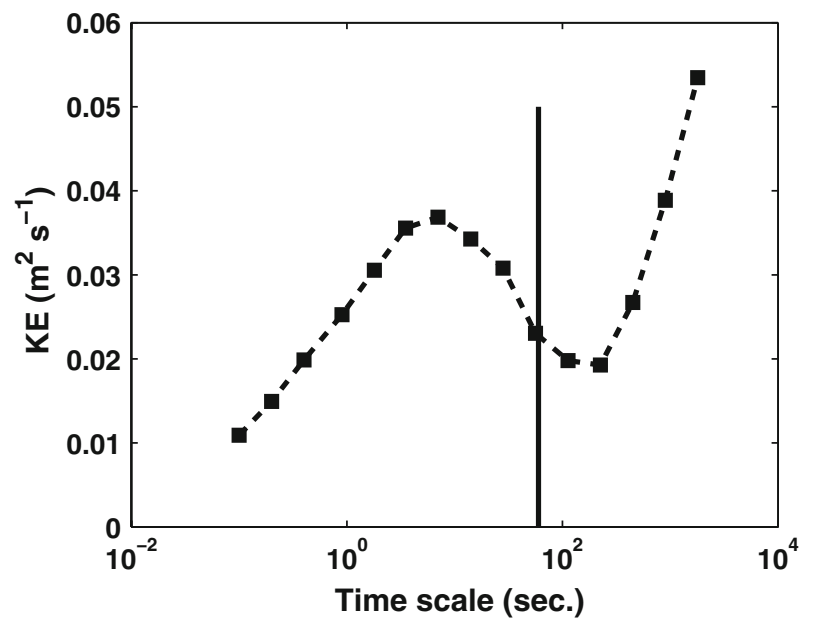

Fig. 1 The scale dependence of the horizontal kinetic energy for the entire turbulence and submeso range of time scales composited over all of the nocturnal records for the Iowa network. The vertical line designates the 1-min time scale used to pragmatically separate turbulence and submeso motions for all of the networks. This study concentrates on submeso scales to the right of the vertical line 


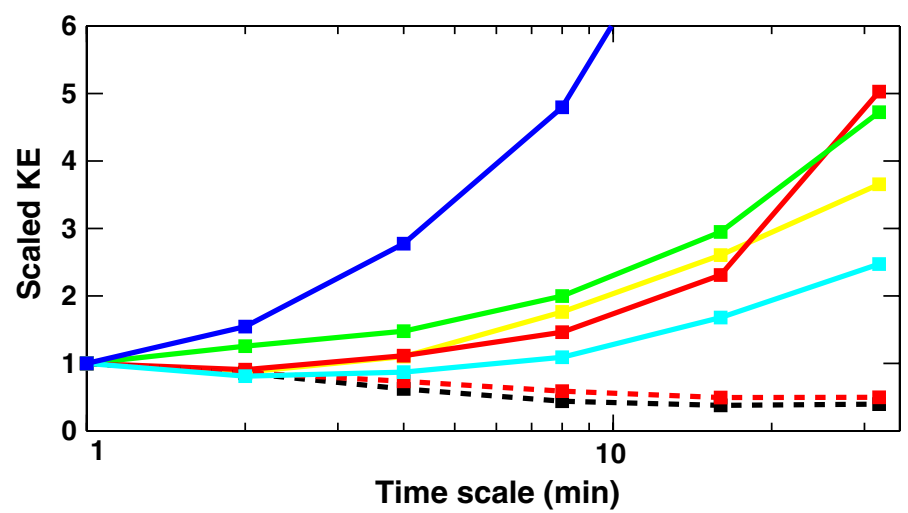

Fig. 2 The horizontal part of the kinetic energy, scaled by the value for the 1-min mode, for Iowa (yellow), CASES99 (red), Hereford (green), EBEX (blue), OHATS (cyan), Young Pine (black dashed) and Mary's River (red dashed), all as a function of time scale ranging from 1 to $32 \mathrm{~min}$

In contrast to the turbulent range, the shape of the spectra for the horizontal kinetic energy in the submeso range varies dramatically between records and shows no tendency to obey spectral similarity theory or maintain a constant spectral slope. Spectra composited over an entire field program (Fig. 2) show a smoother dependence on scale, but show a scaledependent spectral slope at a given site and significant variation between sites.

The spectral slope increases with time scale for the above-canopy networks (Fig. 2), where the increase is greatest for EBEX and least for the OHATS data over the sea. The latter suggests that larger-scale mesoscale motions are less active over the sea although generalizations must await additional datasets. The subcanopy horizontal kinetic energy decreases with scale within the canopy, perhaps due to suppression of larger mesoscales by the overstorey.

The 1-h values of the horizontal kinetic energy for adjacent time scales are highly correlated, but the correlation of horizontal kinetic energy for more separated time scales is small (Fig. 3). Significant sampling errors for individual records contribute to the scatter. The much weaker correlation for more separated time scales reflects the failure of a systematic dependence of spectra on scale for individual records. In contrast, with spectral similarity corresponding to constant spectral slope, the kinetic energy for the different scales would be highly correlated within the scale range of approximate compliance with spectral similarity. Rapid decorrelation of kinetic energy with increasing scale separation is also found for the other networks.

In general, the horizontal kinetic energy for submeso flow is poorly related to other local parameters such as wind speed and stability, as also found by Anfossi et al. (2005) and Vickers and Mahrt (2007). While the kinetic energy may depend on wind speed for a specific scale range and network, no general behaviour could be found. The distribution of horizontal kinetic energy between along-wind and crosswind velocity components is more sensitive to wind speed, albeit with significant dependence on site and time scale. Here, along-wind is defined with respect to the network-averaged, hour-averaged wind vector. For the Iowa network, the velocity variance for the 1-min time scale increases with wind speed (Fig. 4, solid lines) particularly for the along-wind component (black solid). For the 8-min mode, the velocity variance decreases with wind speed, particularly for the crosswind velocity component (red dashed). The shift of energy from larger time scales to smaller time scales and reduction of spectral slope with increasing wind speed is not a sufficient condition for compliance 


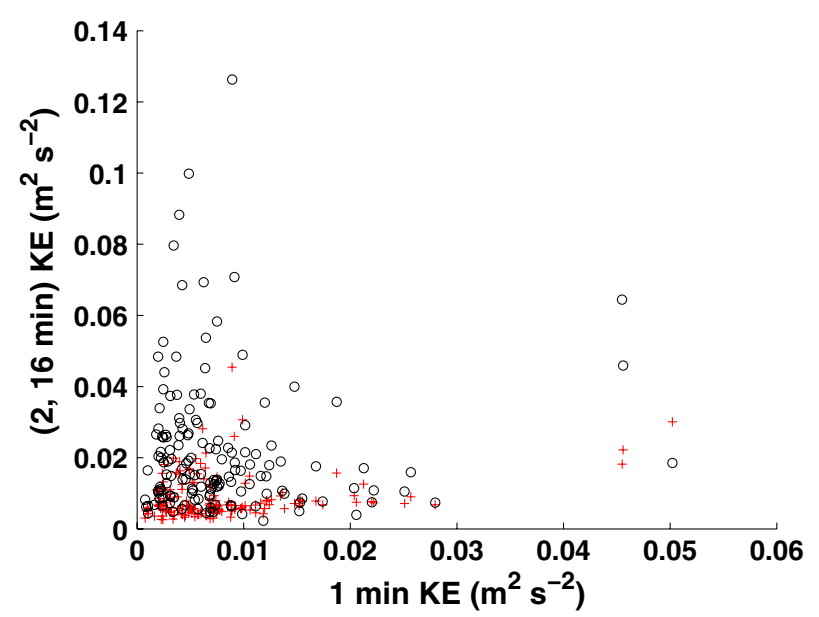

Fig. 3 The relationship between the 1-h values of the 1- and 2-min modes (red plusses) and the relationship between the 1- and 16-min modes (black circles) for the Iowa network

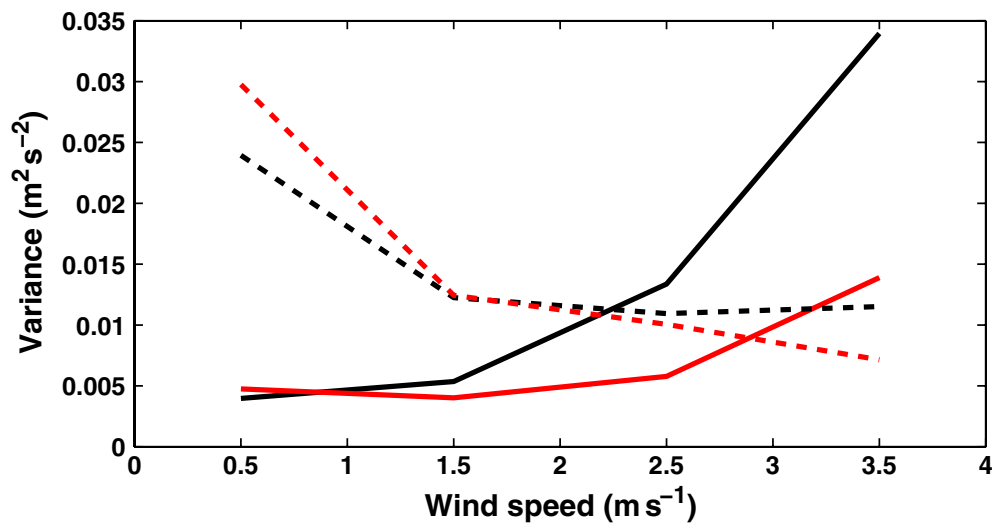

Fig. 4 The averaged along-wind (black) and crosswind (red) velocity variance as a function of wind speed for the 1-min time scale (solid) and the 8-min time scale (dashed) for the Iowa network

with Taylor's hypothesis, which has been rejected based on the weak relationship between horizontal structure and time variability (Mahrt et al. 2008). This shift of energy to smaller scales might be related to stronger shear instability with stronger wind speeds. This scale shift occurs for all of the networks except for the Hereford network where no dependence on wind speed is found, and the Utah network where the spectra steepens with increasing wind speed.

Although one cannot infer horizontal structure from the time spectra, greater variance of the along-wind velocity would be expected for transverse modes such as shear-driven overturning or gravity waves with phase lines perpendicular to the wind speed. Conversely, more crosswind velocity variance might be expected with elongation of the structures in the alongwind direction. Figures 4 and 5 collectively indicate that for the Iowa network, along-wind 

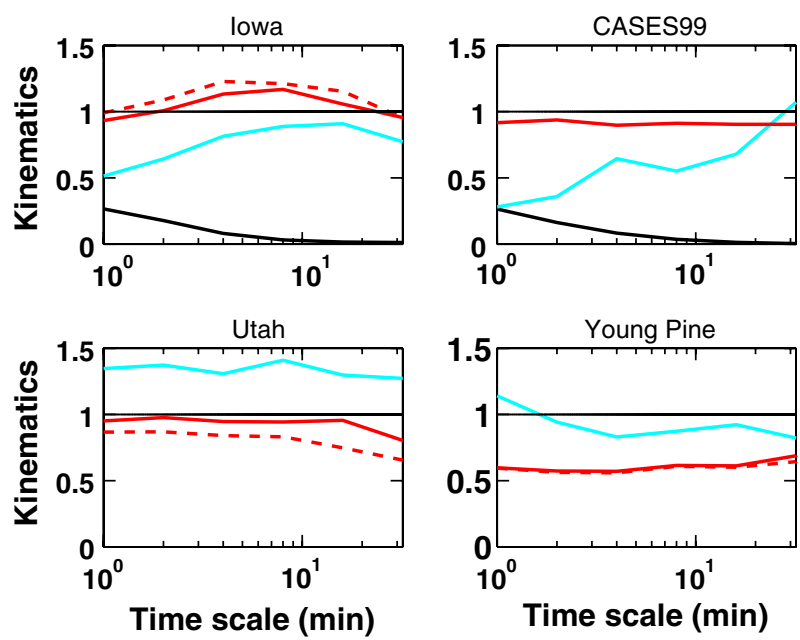

Fig. 5 The ratio of the vertical velocity variance to the horizontal velocity variance (black), the ratio of the crosswind to the along-wind velocity variance (cyan) and the ratio of the vertical vorticity to the horizontal divergence (red, dashed for the larger domain), all as a function of time scale ranging from 1 to $32 \mathrm{~min}$. Domain sizes and characteristics are listed in Table 1

velocity variance is significantly larger than crosswind variance, particularly for stronger winds and smaller time scale. However, the dependence of the relative magnitudes of the along-wind and crosswind velocity variances on wind speed is different for each network. The dependence on time scale is also different for each network (Fig. 5). The significant variation of the structure of the submeso-scale motions between networks will be found throughout this study and is presumably due to different surface conditions and prevailing synoptic flow.

\section{Kinematics of the Horizontal Structure}

The unavailability of network observations at multiple levels and the uncertainty of the measured vertical motion at larger scales precludes meaningful analysis of the three-dimensional dynamics of the submeso-scale structures and also precludes more rigorous analysis of the kinematics, such as presented by Chong et al. (1990) and applied to model output by Gaudet and Cotton (2006). The network data do provide for simple analysis of the kinematics of the horizontal flow. The Iowa, CASES99, Utah and Young Pine networks have suitable station configuration to estimate horizontal gradients required for the kinematical quantities, evaluated below.

Animations of the spatial structure of the wind field based on these networks sometimes reveal well-defined structures with wave-like variations, significant flow curvature and convergence zones. However, the flow more often appears to be a complex melange. While substantial flow curvature in the horizontal plane is often observed, well-defined vortices are uncommon. Most vortex-like motions do not survive a full circulation time scale. Cases with large vertical vorticity are more associated with tangential shear than flow curvature, at least within the ability of the network to resolve such vortices. We now seek a statistical description of the kinematics. 


\subsection{Measure of Horizontal Variability}

As a measure of the spatial variability of each time scale, $p$, we evaluate for each record

$$
V_{\text {Domain }}(p) \equiv<\left(\tilde{u}_{k, p}^{2}+\tilde{v}_{k, p}^{2}\right)^{0.5}>
$$

where the tilde represents the deviation of the wind for station $k$ from the average over all of the stations in the network and the angle brackets indicate averaging over the stations. The spatial variability for each time scale does not show a definable dependence on wind speed, except for the largest time scale of 64 min (Fig. 6), which can include important horizontal variability due to surface heterogeneity. Perhaps the amplitude of the stationary spatial variability due to surface heterogeneity increases with increasing wind speed. Since the 64-min mode includes the stationary effect of surface heterogeneity, its horizontal variation is generally larger than that of the other modes. Smaller scale modes are temporal deviations from the stationary pattern and presumably less influenced by surface heterogeneity and consequently less sensitive to the wind speed (Fig. 6).

For the Iowa and Young Pine networks, $V_{\text {Domain }}$ for the largest time scale increases linearly with wind speed (Fig. 6). Apparently, surface features generate larger amplitude perturbations with stronger wind speed. In contrast, the spatial variability of the record averages for the CASES99 network shows weak dependence on wind speed (Fig. 6), evidently due to the weak surface heterogeneity for this network. The spatial variability of the record averages for the larger heterogeneous Utah network is larger than the values for the other network, but shows minimal increase with wind speed. For this arid network, the spatial variability of the flow is significantly influenced by buoyancy-driven slope flows and the relative role of the large-scale flow may be less.

The transient part of the horizontal variability (less than 1-h time scales) is less related to wind speed. In addition, the transient horizontal variability $\left(V_{\text {Domain }}\right)$ is generally not well correlated to the time variability (not shown), nor is it related to time scale in a systematic way for the collection of networks as a whole (Fig. 6). Submeso flows are complex. The Young
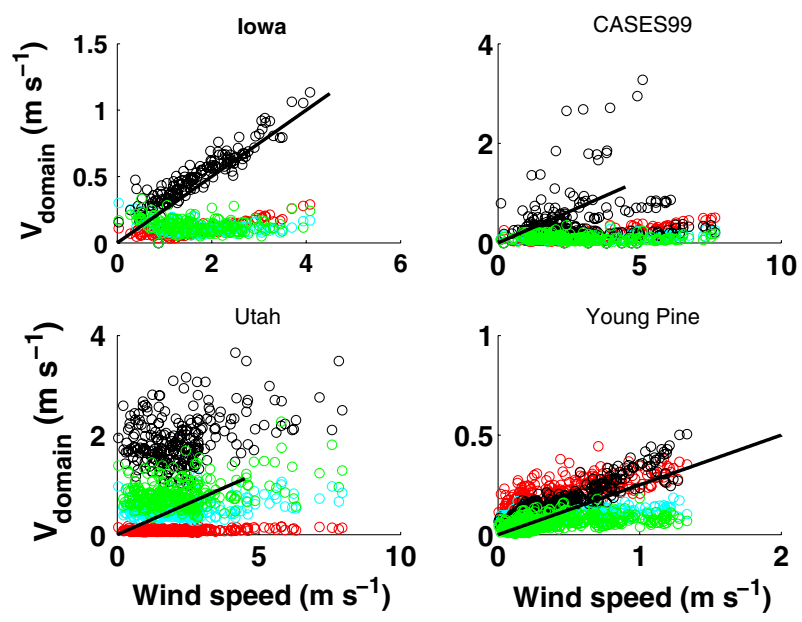

Fig. 6 The horizontal variability $\left(V_{\text {Domain }}\right)$ for the 1-min time scale (red), 8-min time scale (cyan), 32-min time scale (green) and 64-min time scale (black), as a function of the speed of the network averaged wind. The 64-min mode by definition includes spatial variability due to surface heterogeneity (see text). The solid black line represents $0.25 \times$ wind speed. The axis scales are different for each site 
Pine network is an exception in that the horizontal variation of the motion on all time scales increases with wind speed. Evidently, the upstream roughness change induces horizontal variation across this small network that is modulated by time variation of the upstream wind. In particular, the 1-min mode for the Young Pine network (red, Fig. 6) is characterized by significant spatial variation that increases with wind speed at a rate greater than the increase for the 8- and 32-min modes and comparable to the 64-min mode. The Young Pine network is subcanopy and contains relatively more kinetic energy at the smaller time scales compared to the larger time scales (Fig. 2).

\subsection{Scale Dependence of Kinematic Quantities}

The horizontal variability can be partitioned by horizontally expanding about a reference point in terms of a Taylor expansion. The first-order part of this expansion generates the horizontal divergence, the vertical vorticity and the deformation (e.g., Dutton 1986)

$$
\begin{gathered}
\text { Div } \equiv \frac{\partial u}{\partial x}+\frac{\partial v}{\partial y}, \\
\text { Vor } \equiv \frac{\partial v}{\partial x}-\frac{\partial u}{\partial y}, \\
D e f \equiv \frac{\partial v}{\partial x}+\frac{\partial u}{\partial y}+\frac{\partial u}{\partial x}-\frac{\partial v}{\partial y} .
\end{gathered}
$$

These quantities are computed for the four networks according to Sect. 2.2. The dependence of the gradients on time scale must be interpreted with caution since the station spacing acts as a low pass spatial filter. Spatial scales comparable to the station spacing, or smaller, are not captured. Since these smaller horizontal scales tend to correspond to smaller time scales, the finite station spacing also acts as a low pass time filter with unknown response function. Therefore the estimated dependence of the kinematic quantities on time scale can be considered only qualitatively.

For the Iowa domain, the kinematic quantities are computed using an 1,800 m separation distance (the "small grid") and 5,400 m separation distance ("large grid"). The flow for the largest time scale $(64 \mathrm{~min})$ includes the mean, presumably associated with surface heterogeneity, and corresponds to the largest kinematic quantities after averaging over the field program. For the smaller time scales, the vertical vorticity and divergence both approach zero when averaged over the entire dataset since there is no obvious preferred sign. Therefore, we average absolute values. The averages are computed independently for each multiresolution time scale. For such averages, the vorticity and divergence do not show a systematic dependence on time scale amongst the different networks (Fig. 5).

Here, we form a nondimensional quantity as the ratio of the vertical vorticity to the horizontal divergence. This ratio is theoretically small for simple two-dimensional gravity waves and large for approximately two-dimensional modes such as pancake vortices. Both transverse and longitudinal modes contribute to horizontal divergence, but do not lead to vertical vorticity without tilting or secondary three-dimensional instabilities. In contrast, vortex merging with kinetic energy transfer to larger scales would enhance the vorticity at larger scales, discussed in Riley and Lelong (2000) and McWilliams (2004). The above expectations collectively predict that the ratio of vertical vorticity to horizontal divergence increases with increasing horizontal scale. 
Except for the Young Pine network, discussed further below, the ratio of the vorticity to the divergence is order of unity (Fig. 5). Since the ratio of the vertical vorticity to the horizontal divergence does not change appreciably with time scale and the flow animations indicate that well-defined vortices are uncommon or masked by other processes, we conclude that vortices in the horizontal plane are not a dominating influence in the present data. More definite conclusions must await more dense networks. Measurements of vertical structure would be required to examine the interaction between gravity waves and two-dimensional vorticies, as observed in laboratory flows (Godoy-Diana et al. 2006).

For the smaller CASES99 domain (Fig. 5), where the derivatives are computed over a 400-m separation distance, the ratio of the vorticity to the divergence is also order of unity. For the large Utah domain, the small grid separation is $7 \mathrm{~km}$ and the large grid spacing is $21 \mathrm{~km}$. The ratio of the vertical vorticity to the horizontal divergence is slightly less than one and decreases slightly with horizontal scale.

For the small Young Pine network, the gradients are computed using 40-m separation distance (small grid) and 120-m separation distance (large grid). The ratio of vorticity to horizontal divergence increases slightly with increasing time scale, but remains less than 0.7. A large decrease of surface roughness immediately upwind from the network leads to flow acceleration and enhanced horizontal divergence for this network. Except for this network, the ratio of vorticity and divergence is generally order of unity and this ratio is less variable between networks compared to the ratio of crosswind to along wind velocity variances.

\subsection{Scale Dependence Based on Different Networks}

Assessment of the dependence on horizontal scale, based on the different sized networks, is contaminated by different surface conditions, topography and prevailing synoptic conditions. The flow statistics also depend on network size and station configuration, as can be inferred from Belušić and Mahrt (2008). Nonetheless, the absolute value of vorticity and divergence decrease within increasing horizontal scale by an order of magnitude, shown in Fig. 7 for the 16-min mode. This trend is not sensitive to the choice of time scale. The larger values at smaller horizontal scales could be partly due to gradient errors associated with smaller differences between larger numbers. The difference between vorticity and divergence at the smallest horizontal scales is due to the upstream roughness change associated with the two grids at the Young Pine network. Nonetheless, Fig. 7 is consistent with the decrease of gradients with increasing horizontal scale. It is not known why the averaged absolute values of vorticity and divergence are numerically close. The vorticity and divergence for individual 1-h records are poorly correlated. One might be tempted to define the scale division between submeso and mesoscale motions as the decreased scale dependence beginning at a horizontal scale of a few kilometres. However, this argument can be confidently pursued only when the entire scale range is provided by a single network.

Although comparable calculations are not available for turbulence scales, the CASES99 network captures the vorticity and divergence of the convective eddies in the daytime, which is about an order of magnitude greater than the nocturnal submeso motions at the same horizontal scales.

\subsection{Kinematic Quantities for Individual Records}

The correlations between different kinematic quantities are generally weak. The most significant correlations involve deformation, which is partly due to the fact that the deformation 


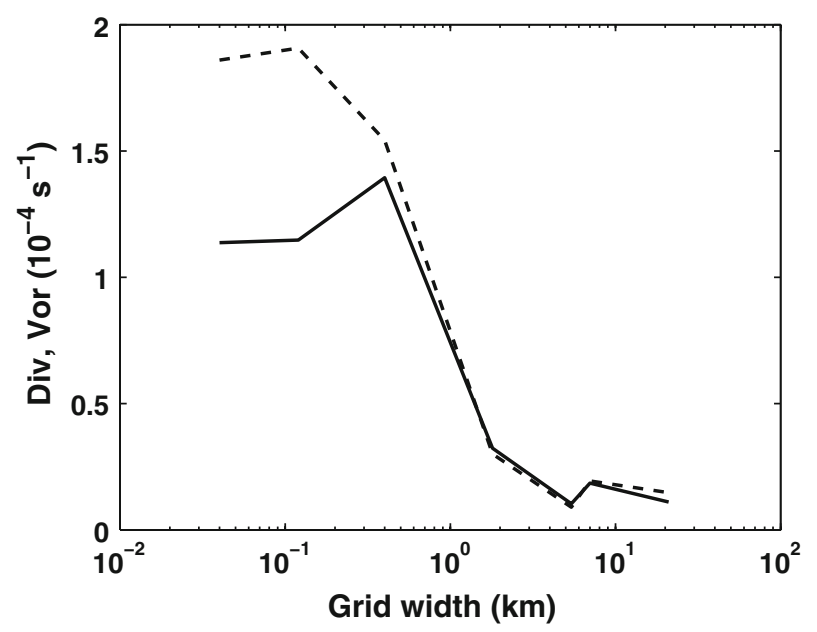

Fig. 7 The averaged absolute value of the vertical vorticity (solid) and horizontal divergence (dashed) for the 16-min mode as a function of horizontal scale of the calculation of the gradients. The two smallest scales are the Young Pine network where an upstream roughness decrease leads to acceleration and enhanced horizontal divergence. The two largest horizontal scales are from the Utah network with complex topography

shares variables with the divergence and with the vorticity, leading to self-correlation. For example, large between-record variation of $\partial u / \partial x$ leads to positive correlation between divergence and deformation. Large variation of $\partial v / \partial y$ leads to negative correlation between divergence and deformation.

Cases with large deformation and vorticity of the same sign typically resulted from large $\partial v / \partial x$ associated with a sign switch in the $v$-component between the western and eastern parts of the Iowa network. The deformation and vorticity are strong negatively correlated for the smaller scale Utah grid as a result of significant between-record variation of $\partial u / \partial y$. This is sometimes associated with a north-easterly component in the northern part of the network associated with cold air drainage, or a south-easterly component associated with barrier winds in the eastern part of the network induced by the Cedar Mountains. While the correlation between kinematic quantities may show scale dependence for a given network, this scale dependence is significantly different for each network.

\section{Gradient Kurtosis}

The stratified boundary layer is occupied by numerous isolated events (e.g., Anderson 2003; Sun et al. 2004; Mahrt 2008). Here, wind changes will be summarized in terms of kurtosis of the velocity components for each time scale, as shown for the Iowa network in Fig. 8 (solid lines). The kurtosis is large at the smallest time scales, about 7 for the 1-min mode, and decreases with increasing time scale to a value of about 3 for the largest time scale. The frequency distribution for the smaller time scales is characterized by some velocity fluctuations of particularly large magnitude of either sign. This leads to longer tails in the frequency distribution of the velocity components compared to a Guassian distribution. Recall that the 1 -min mode is the deviations of the 1-min velocity components from the 2-min averages, the 2-min mode is the deviation of the 2-min averages from 4-min averages and so forth. 


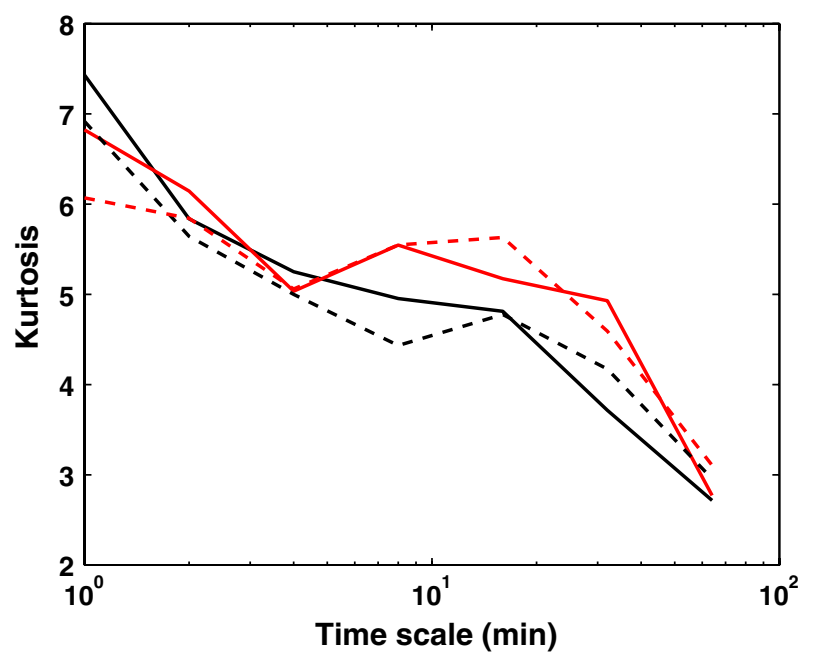

Fig. 8 The kurtosis of the velocity components $(\mathrm{u}=$ solid black, $v=$ solid red $)$ averaged over all of the stations in the Iowa network as a function of time scale ranging from 1 to 32 minutes. The kurtosis of two-station velocity differences averaged over all possible station pairs as a function of time scale (dashed lines)

The two-point velocity differences between pairs of stations are also characterized by kurtosis significantly greater than 3 at the smaller time scales. That is, horizontal variations of the velocity components are characterized by occasional large magnitudes of either sign, perhaps corresponding to a submeso front between the two stations. The kurtosis of the horizontal variations shows little dependence on the magnitude of the separation distance between the stations. Averaged over all of the different two-station combinations in the Iowa network, the kurtosis of the horizontal velocity differences (Fig. 8, dashed lines) decreases with time scale in a manner similar to the decrease of the kurtosis of time changes with increasing time scale.

Both time changes and horizontal variations of the velocity components are characterized by near zero skewness; there is no preferred sign to the velocity changes with respect to time or horizontal distance. The kurtosis of the wind variations for the other networks is either characterized by similar behaviour, or, larger values of kurtosis and less systematic dependence on time scale. Based on the behaviour of the kurtosis of the wind component variations, we conclude that submeso motions are significantly non-Gaussian. These results suggest that sharp changes in the wind field are important in both the time domain and the space domain.

\section{Conclusions}

Eight networks of anemometer data were selected to study the horizontal structure of submeso motions in the stable boundary layer and the dependence of this horizontal structure on time scale. While the submeso spectra for individual records are erratic, the spectra averaged over the entire field program show a more organized dependence on scale. However, such spectra are site dependent and the spectral slope generally increases with time scale, precluding application of similarity. In this regard, submeso spectra in the boundary layer 
are more complex than mesoscale spectra in the upper troposphere and more complex than turbulence spectra in the boundary layer.

The horizontal variation has been divided into time averages over each record (approximately $1 \mathrm{~h}$ ) and time deviations from the record averages, referred to as the transient part. The record average horizontal variation includes the influence of surface heterogeneity. This "stationary" contribution to the horizontal variation increases with wind speed, although the degree and form of this dependence varies between networks. This study concentrated on the transient part of the horizontal variation, which showed little dependence on wind speed.

Animations of the wind field reveal a wide variety of complex time and space structures, sometimes wave-like. The wide variety of complex submeso signatures is not amenable to classification, and therefore we examined the stochastic behaviour of the submeso motions. For example, the average magnitude of the vertical vorticity and horizontal divergence decreases with increasing horizontal scale. However, the ratio of the vertical vorticity to the horizontal divergence generally remains order of one for the space and time scales examined, except for one small network where a roughness change forces stronger horizontal divergence. The predicted increase of this ratio with increasing scale is not observed. Although the vorticity/divergence ratio does not show a systematic dependence on horizontal scale, the physics presumably does vary with scale. This scale dependence can be inferred, for example, from the decreasing kurtosis of the velocity variations with increasing scale and decreasing ratio of vertical velocity variance to horizontal velocity variance with increasing scale.

The events with the largest vorticity and deformation are mainly associated with acceleration of the flow in part of the network; i.e., the largest values of vorticity are more associated with lateral shear than curvature of the streamlines, although partial vortices are sometimes observed. The present data do not reveal significant two-dimensional vortices (pancake vorticies), although limitations of the spatial resolution prevent definite conclusions. The kurtosis of time changes and horizontal changes of the velocity components are characterized by large values, greater than 5 for time scales less than $5 \mathrm{~min}$, suggesting non-Gaussian, event-like behaviour.

The present study failed to reveal the dynamics of complex submeso-scale flows. The networks used in this study were not designed for the present analysis and impose limitations on the conclusions. A denser and more extensive network with more systematic station spacing and more extensive information on vertical structure would be needed.

Acknowledgements The helpful comments of the reviewers are greatly appreciated. The EBEX and CASES99 data were provided by the Integrated Surface Flux Facility of the National Center for Atmospheric Research. This material is based upon work supported by NSF Grant ATM-0607842, DTRA grant W911NF-06-1-0439 and ARO Contract W911FN05C0067.

Open Access This article is distributed under the terms of the Creative Commons Attribution Noncommercial License which permits any noncommercial use, distribution, and reproduction in any medium, provided the original author(s) and source are credited.

\section{References}

Anderson PS (2003) Fine-scale structure observed in a stable atmospheric boundary layer by Sodar and kiteborne tethersonde. Boundary-Layer Meteorol 107:323-351

Anfossi D, Oettl D, Degrazia G, Boulart A (2005) An analysis of sonic anemometer observations in low wind speed conditions. Boundary-Layer Meteorol 114:179-203 
Anquetin A, Guilbaud C, Chollet J-P (1998) The formation and destruction of inversion layers within a deep valley. J Appl Meteorol 37:1547-1560

Belušić D, Mahrt L (2008) Estimation of length scales from mesoscale networks. Tellus 60:706-715

Chong M, Perry A, Cantwell B (1990) A general classification of three-dimensional flow fields. Phys Fluids 2:765-777

Dutton JA (1986) The ceaseless wind. Dover, New York, $671 \mathrm{pp}$

Fox-Kemper B, Ferrari R, Hallberg R (2008) Parameterization of mixed layer eddies. Part I: theory and diagnosis. J Phys Oceanogr 38:1145-1165

Gaudet B, Cotton W (2006) Low-level mesocyclonic concentration by non-axisymmetric transport. Part I: supercell and mesocyclone evolution. J Atmos Sci 63:1113-1133

Godoy-Diana R, Chomaz J-M, Donnadieu C (2006) Internal gravity waves in a dipolar wind: a wave-vortex interaction experiment in a stratified fluid. J Fluid Mech 548:281-308

Hanna S, Chang J (1992) Representativeness of wind measurements on a mesoscale grid with station separations of $312 \mathrm{~m}$ to $10 \mathrm{~km}$. Boundary-Layer Meteorol 60:309-324

Howell J, Mahrt L (1997) Multiresolution flux decomposition. Boundary-Layer Meteorol 83:117-137

Kustas W, Li F, Jackson J, Preuger J, MacPherson J, Wolde M (2004) Effects of remote sensing pixel resolution on modeled energy flux variability of croplands in Iowa. Remote Sens Environ 92:535-547

Mahrt L (2007) Weak-wind mesoscale meandering in the nocturnal boundary layer. Environ Fluid Mech 7:331-347

Mahrt L (2008) Mesoscale wind direction shifts in the stable boundary-layer. Tellus 60A:700-705

Mahrt L, Thomas C, Preuger J (2008) Mesoscale wind direction shifts in the stable boundary-layer. Q J Roy Meteorol Soc (in press)

McWilliams J (2004) Phenomenological hunts in two-dimensional and stably stratified turbulence. In: Federovich E, Rotunno R, Stevens B (eds) Atmospheric turbulence and mesoscale meteorology. Cambridge University Press, pp 35-49

Mestayer PG, Anquetin S (1995) Climatology of cities. In: Gyr A, Rys F-S (eds) Diffusion and transport of pollutants in atmospheric mesoscale flow fields. Kluwer Academic Publishers, The Netherlands, pp 165-189

Meunier P, Le Dizès S, Leweke T (2005) Physics of vortex merging. C R Physique 6:431-450

Monti P, Fernando H, Chan W, Princevac M, Kowalewski T, Pardyjak E (2002) Observations of flow and turbulence in the nocturnal boundary layer over a slope. J Atmos Sci 59:2513-2434

Nappo CJ (2002) An introduction to atmospheric gravity waves. Academic Press, Boston, 297 pp

Oncley SP, Foken T, Vogt R, Kohsiek W, De Bruin H, Bernhofer C, Christen A, Van Gorsel E, Grantz D, Lehner I, Liebethal C, Liu H, Mauder M, Pitacco A, Ribeiro L, Weidinger T (2007) The energy balance experiment EBEX-2000. Part I: overview and energy balance. Boundary-Layer Meteorol 123:1-28

Poulos G, Blumen W, Fritts D, Lundquist J, Sun J, Burns S, Nappo C, Banta R, Newsom R, Cuxart J, Terradellas E, Balsley B, Jensen M (2001) CASES-99: a comprehensive investigation of the stable nocturnal boundary layer. Bull Am Meteorol Soc 83:555-581

Riley JJ, Lelong M-P (2000) Fluid motions in the presence of strong stable stratification. Ann Rev Fluid Mech 32:613-657

Schwarz P, Law B, Williams M, Irvine J, Kurpius M, Moore D (2004) Climatic versus biotic constraints on carbon and water fluxes in seasonally drought-affected ponderosa pine ecosystems. Glob Biochem Cycles 18:1029-1037

Steeneveld G, Holtslag AAM, Nappo CJ, van de Wiel BJH, Mahrt L (2008) Exploring the possible role of small scale terrain drag on stable boundary layers over land. J Clim Appl Meteorol 47:2518-2530

Sullivan PP, Edson J, Horst TW, Wyngaard JC, Kelly M (2006) Subfilter scale fluxes in the marine surface layer: results from the ocean horizontal array turbulence study (OHATS). 17th symposium on boundary layers and turbulence. Am Met Soc http://ams.confex.com/ams/BLTAgFBioA/techprogram/paper_ 110884.htm

Sun J, Lenschow DH, Burns SP, Banta RM, Newsom RK, Coulter R, Frasier S, Ince T, Nappo C, Balsley B, Jensen M, Mahrt L, Miller D, Skelly B (2004) Atmospheric disturbances that generate intermittent turbulence in nocturnal boundary layers. Boundary-Layer Meteorol 110:255-279

Thomas C, Martin JG, Goeckede M, Siqueira MB, Foken T, Law B, Loescher H, Katul G (2008) Estimating daytime subcanopy respiration from conditional sampling methods applied to multi-scalar high frequency turbulence time series. Ag For Meteorol 148:1210-1229

Vickers D, Mahrt L (2006) A solution for flux contamination by mesoscale motions with very weak turbulence. Boundary-Layer Meteorol 118:431-447

Vickers D, Mahrt L (2007) Observations of the cross-wind velocity variance in the stable boundary layer. Environ Fluid Mech 7:55-71

Waite M, Bartello P (2004) Stratified turbulence dominated by vortical motion. J Fluid Mech 517:281-308 